\title{
SnapshotNIR: a handheld multispectral imaging system for tissue viability assessment
}

\section{Michael Sowa}

Michael G. Sowa, "SnapshotNIR: a handheld multispectral imaging system for tissue viability assessment," Proc. SPIE 11144, Photonics and Education in Measurement Science 2019, 111440B (17 September 2019); doi:

$10.1117 / 12.2534989$

SPIE Event: Joint TC1 - TC2 International Symposium on Photonics and Education in Measurement Science 2019, 2019, Jena, Germany 


\title{
SnapshotNIR, a handheld multispectral imaging system for tissue viability assessment.
}

\author{
Michael G. Sowa \\ Kent Imaging Inc., 804B 16 Ave SW, Calgary, Alberta, Canada T2R 0S9
}

\begin{abstract}
Tissue survival depends on the hemoglobin in blood to deliver oxygen to support cellular respiration. Without oxygenated blood surrounding tissue dies. Oxygenated hemoglobin has a distinct visible - near infrared absorption spectrum compared to deoxygenated hemoglobin. A hand-held multispectral reflectance imaging device, SnapshotNIR, was designed to provide a measure of the relative attenuation of reflected light from oxygenated and deoxygenated hemoglobin. The device combines the use of near real-time image classification / segmentation and regression to effectively determine the ratio of oxygenated to deoxygenated hemoglobin in the superficial vascular bed being imaged. This measurement of hemoglobin oxygen saturation can help detect local deficits in oxygen delivery to tissue. This simple, hand-held, battery-powered imaging device can quickly survey large areas of tissue in a complete non-invasive fashion. It is easy to use and suitable for use in the operating room or clinics that have limited infrastructure. Examples are given on the performance of the device in general surgery and in the assessment of peripheral circulation.
\end{abstract}

Keywords: multispectral imaging, hemoglobin oxygenation, reconstructive surgery, chronic wound assessment, image processing

\section{INTRODUCTION}

The visible and short wavelength near infrared regions of the electromagnetic spectrum have a long history of being used to provide a measure of hemoglobin oxygen saturation. ${ }^{1}$ When the measurement is made in-vivo, the short wavelength near infrared region is usually preferred because of its ability to penetrate more deeply into tissue. This becomes particularly important when trying to make trans-cutaneous measurements. Epidermal melanin, especially in darker skin, blocks much of the shorter wavelength visible light from sampling the blood supply that resides in the dermal layer of the skin, beneath the epidermis. Pulse oximetry, one of the best known and most impactful optical measurement devices in medicine, illuminates skin with deep red and near infrared light and measures the reflected or transmitted light. Hemoglobin which is the dominate oxygen carrier in the body absorbs red - near infrared light differently when it is carrying oxygen, $\mathrm{HbO}_{2}$ from when it is deoxygenated, $\mathrm{Hb}$. Figure 1 plots the differential light absorption of oxygenated and deoxygenated hemoglobin in the red and near infrared spectral regions. By measuring the ratio of red to near infrared reflected or transmitted by tissue as well as making this measurement at the peak (systolic) and trough (diastolic) of the heart pulse, pulse oximetry provides a reliable estimate of the arterial hemoglobin oxygen saturation, invivo. The real-time $\mathrm{S}_{\mathrm{p}} \mathrm{O}_{2}$ measurement of hemoglobin oxygen saturation given by a pulse oximeter provides an immediate assessment of a person's systemic oxygenation status which is indicative of cardiac output and pulmonary function.

There are clinical situations where the measurement of the local hemoglobin oxygenation status is important. For example, in the assessment of peripheral circulation or chronic wounds, areas of tissue not receiving an adequate supply of oxygen will go on to die. Identifying those areas to enable the clinician to try to correct the oxygen deficit or resect the tissue are key steps in the effective management of wounds or the re-establishment of adequate oxygen delivery to the periphery. A simple, noninvasive imaging tool facilities these steps and further helps the clinician to monitor the the effects of their treatment or intervention. There are several technologies, some of them optically based, that are used for wound assessment and monitoring. ${ }^{2}$ However, the ability to survey large areas of tissue in a single image and to acquire that image completely non-invasively in a matter of seconds makes red - near infrared spectral imaging stand-out compared to competing methods. In addition to wound assessment and management, local hemoglobin oxygenation assessment during surgical reconstruction of tissue is important. The near real-time performance coupled the ability to image a large area of tissue make red - near infrared spectral imaging a useful tool for intra-operative, surgical guidance.

*mike@kentimaging.com; phone 1403 455-7610; kentimaging.com

Photonics and Education in Measurement Science 2019, edited by Maik Rosenberger,

Paul-Gerald Dittrich, Bernhard Zagar, Proc. of SPIE Vol. 11144, 111440B

(C) 2019 SPIE · CCC code: 0277-786X/19/\$21 - doi: 10.1117/12.2534989 
We have designed a simple, hand-held, battery powered, red - near infrared multispectral imaging system, SnapshotNIR, specifically for determining the local hemoglobin oxygen saturation in the near - surface tissue vascular beds over a large area of tissue. The device has wide applicability in medicine, particularly in the areas of acute and chronic wound assessment and management as well as plastic and reconstructive surgery.

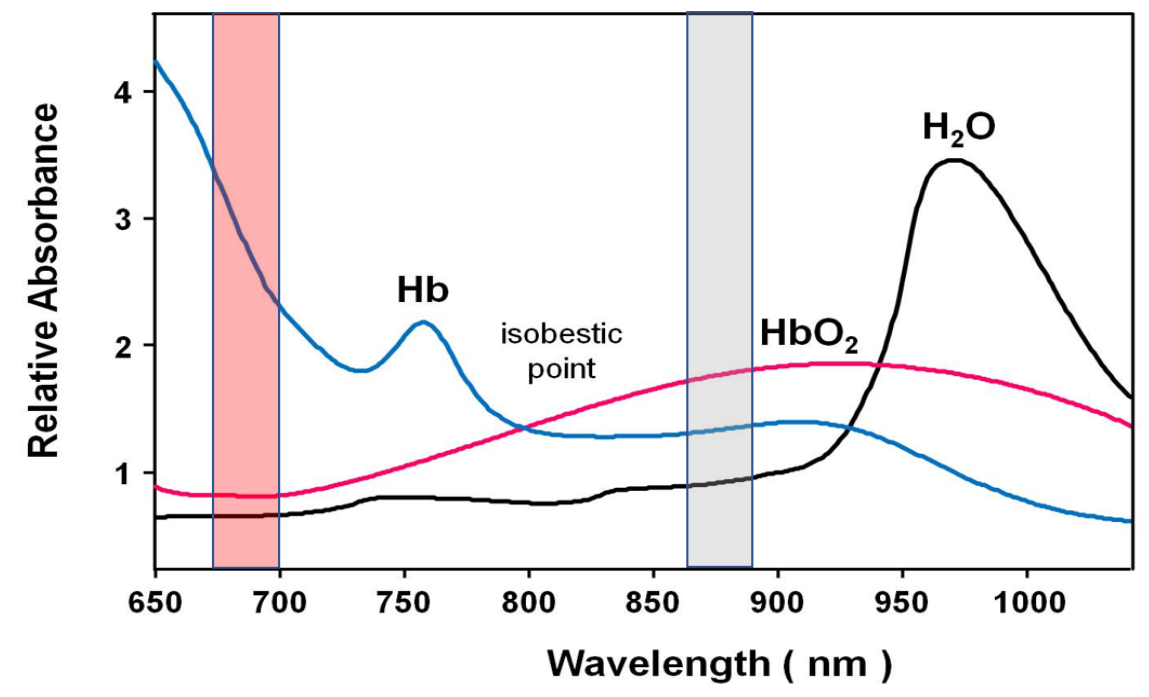

Figure 1. Pulse oximetry measures the reflected or transmitted light from tissue in the deep red and at a longer near-infrared region. The differential light absorption of hemoglobin when carrying oxygen $\left(\mathrm{HbO}_{2}\right)$ and when deoxygenated $(\mathrm{Hb})$ in these two wavelength regions is exploited to determine the relative proportion of $\mathrm{HbO}_{2}$ to $\mathrm{Hb}$ based on the ratio of red to near infrared light that is reflected or transmitted by the tissue. 


\section{DEVICE DESCRIPTION}

The SnapshotNIR device is depicted in Figure 2. It is a handheld reflectance multispectral imaging system designed to be used as a standard digital camera. Multispectral imaging is achieved by acquiring a series of images across a sequence of LED flashes where the LED emission bands span key regions of the red and near infrared range to optimize the measurement of the differential reflectance from oxygenated and deoxygenated hemoglobin (see Figure 1). A linear un-mixing algorithm that incorporates a weak regularization term to add further robustness is used to provide an estimate of the relative concentrations of oxy- $(\mathrm{HbO} 2)$ and deoxy-hemgoblin $(\mathrm{Hb})$. This procedure is described in detail in Sowa and Cervi. ${ }^{3}$ Using the estimated relative concentrations of the oxygenated and deoxygenated forms of hemoglobin, a hemoglobin oxygen saturation is calculated in the standard form, equation 1. Clinical hemoglobin oxygen saturation nomenclature uses $\mathrm{S}_{\mathrm{a}} \mathrm{O}_{2}$ and $\mathrm{S}_{\mathrm{v}} \mathrm{O}_{2}$ to stand for oxygen saturation measurements taken from arterial and venous blood samples. Reflectance imaging measurements of tissue, in-vivo, probe the more superficial vascular beds and in our case is not restricted to either the arterial or venous compartments. We therefore use the notation, $\mathrm{S}_{\mathrm{t}} \mathrm{O}_{2}$, or tissue hemoglobin oxygen saturation, to distinguish our saturation measurement from those that sample the arterial $\left(\mathrm{S}_{\mathrm{a}} \mathrm{O}_{2}\right)$ or venous blood $\left(\mathrm{S}_{\mathrm{v}} \mathrm{O}_{2}\right)$, or $\mathrm{S}_{\mathrm{p}} \mathrm{O}_{2}$ measurements from a pulse oximeter that tend to be weighted to arterial blood hemoglobin oxygenation. Unlike $\mathrm{S}_{\mathrm{a}} \mathrm{O}_{2}, \mathrm{~S}_{\mathrm{v}} \mathrm{O}_{2}$ and to a large extent $\mathrm{S}_{\mathrm{p}} \mathrm{O}_{2}$ which provide important information on the systemic health of the patent, $\mathrm{S}_{\mathrm{t}} \mathrm{O}_{2}$ measurements indicate the local hemoglobin oxygenation status of the tissue at the site of the measurement.

$$
\mathrm{S}_{\mathrm{t}} \mathrm{O}_{2}=\frac{[\mathrm{HbO2]}}{\lceil\mathrm{Hb}\rceil+[\mathrm{HbO2}\rceil}
$$

\section{Snapshort网}

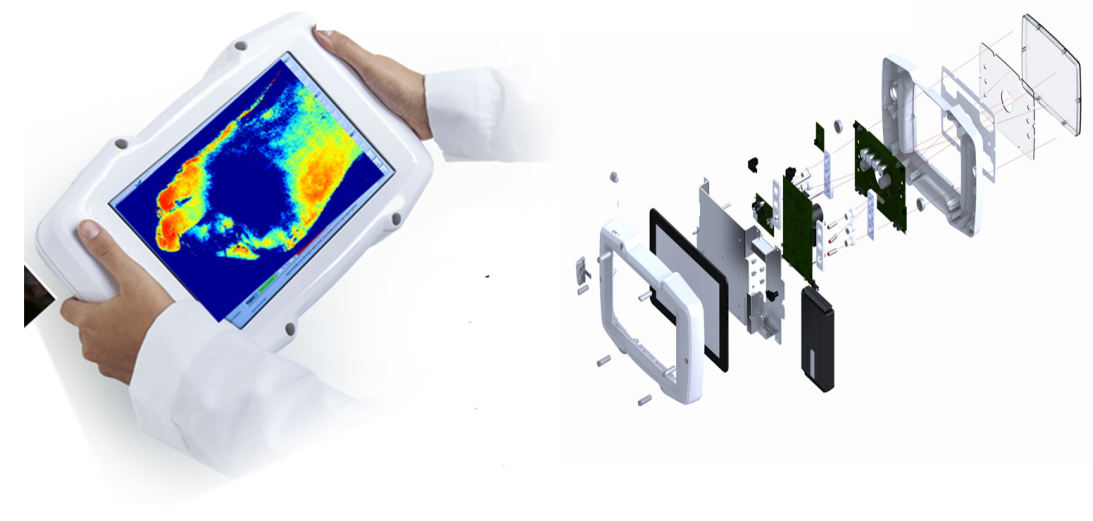

Figure 2. SnapshotNIR is a handheld, battery powered, multispectral imaging system that provides a near real-time measurement of tissue hemoglobin oxygen saturation. 


\section{RESULTS}

\subsection{Validation}

The SnapshotNIR multispectral imaging device was compared to a clinically established technology, indocyanine green (ICG) fluorescence angiography. ICG angiography requires the use of a fluorescent dye, indocyanine green, that gets injected into the blood stream. An expanded laser beam excites the dye to fluoresce while a video camera with an optical band-pass filter set at the peak emission wavelength of the fluorescence of the dye monitors the illuminated area. As the bolus dose of dye rushes through the vascular bed illuminated by the laser, the video camera captures the in-flow and out-flow of the dye. Areas of good blood perfusion show intense fluorescence with rapid in-flow and out-flow kinetics. The Novadaq/Stryker Elite fluorescence imaging system is based on these principles and was used to assess perfusion of tissue in conjunction with the SnapshotNIR multispectral imaging device. The diagnostic performance of the two devices were compared. Tables 1 reports the 95\% confidence interval for the area under the receiver operating characteristics curves (AUC-ROC) when the two devices are used to predict pedicle skin flap survival. Table 2 reports the $95 \%$ confidence interval of the sensitivity of immediate post-operative measurements for predicting viable tissue at 72 -h post-op at fixed specificity of $90 \%$ for predicting 72 -h necrosis. Details of this study can be found in Jones et al. ${ }^{4}$ The study showed that the invasive fluorescent dye based method for assessing local perfusion and non-invasive red near infrared spectral imaging using SnapshotNIR had comparable predictive accuracy in predicting tissue viability in pedicle skin flaps.

Table 1. The $95 \%$ confidence intervals of the area under the receiver operating characteristics curve (AUC) comparing the diagnostic accuracy of predicting tissue viability in pedicle skin flaps of $\mathrm{S}_{\mathrm{t}} \mathrm{O}_{2}$ measurements using the Snapshot device and indocyanine green (ICG) fluorescence angiography using the Novadaq/Stryker SPY Elite device.

\begin{tabular}{|c|c|c|c|}
\hline & $\mathrm{S}_{\mathrm{t}} \mathrm{O}_{2}$ & ICG & $\mathrm{S}_{\mathrm{t}} \mathrm{O}_{2}-$ ICG \\
\hline AUC & {$[0.94,0.96]$} & {$[0.92,0.94]$} & {$[0.01,0.04]$} \\
\hline
\end{tabular}

Table 2. Comparison of the sensitivity of $\mathrm{S}_{\mathrm{t}} \mathrm{O}_{2}$ measurements using the Snapshot device and indocyanine green (ICG) fluorescence angiography using the Novadaq/Stryker SPY Elite device in predicting pedicle skin flap viability when the specificity for detecting necrosis is fixed at $90 \%$. The $95 \%$ percentile bootstrap confidence intervals are reported for the sensitivity (SE) when the specificity (SP) is fixed at $90 \%$.

\begin{tabular}{|c|c|c|c|}
\hline & $\mathrm{S}_{\mathrm{t}} \mathrm{O}_{2}$ & ICG & $\mathrm{S}_{\mathrm{t}} \mathrm{O}_{2}-$ ICG \\
\hline $\mathrm{SE} @ \mathrm{SP}=90 \%$ & {$[86.31,91.02]$} & {$[79.06,86.85]$} & {$[1.57,9.85]$} \\
\hline
\end{tabular}

\subsection{Example from general surgery}

The real-time imaging capability combined with the usability of a digital camera make red - near infrared spectral imaging convenient enough to use intra-operative without a major disruption to surgical workflow. The left panel of Figure 3 shows an RGB image of an exposed bowel with suspected areas of poor blood supply. The right panel shows the corresponding Snapshot $\mathrm{S}_{\mathrm{t}} \mathrm{O}_{2}$ image. The tissue hemoglobin oxygenation image is color coded where red indicates 
high local hemoglobin oxygenation while blue indicates low oxygenation. With the $\mathrm{S}_{t} \mathrm{O}_{2}$ image the surgeon can more easily locate areas of concern and deal with those areas. The ability to re-image without having to inject a dye, means that the surgeon can image repeatedly throughout the surgery to follow progress.
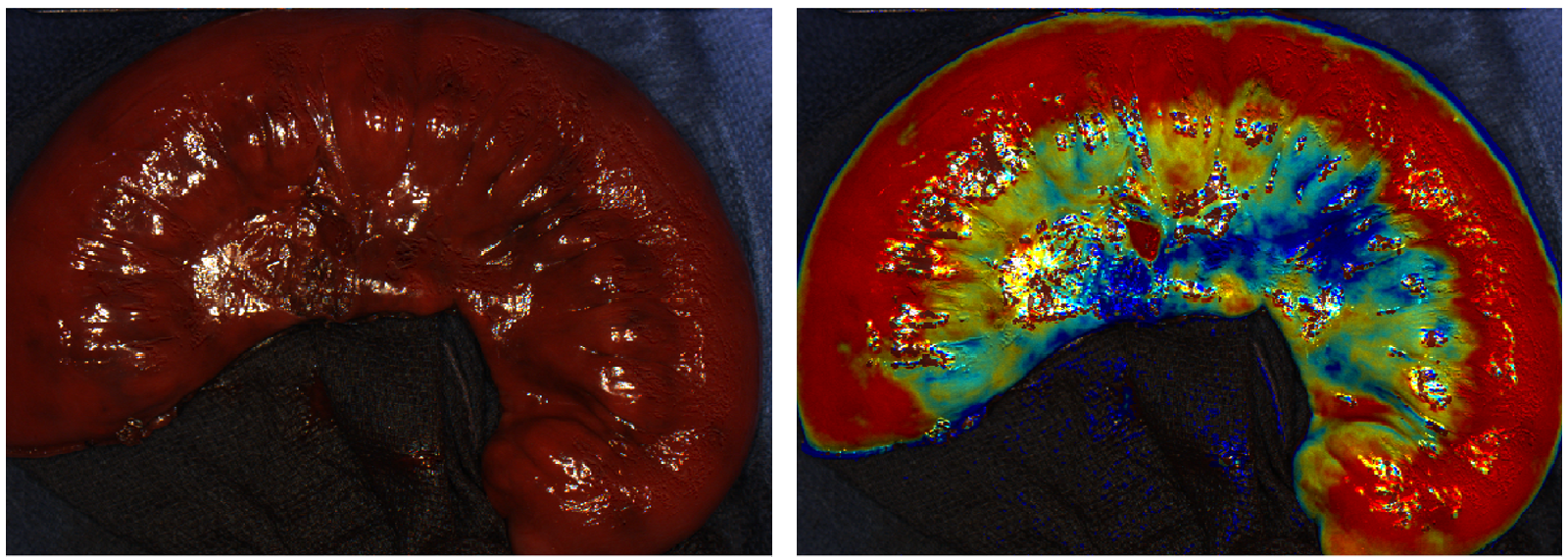

Figure 3. SnapshotNIR can be used intra-operatively to locate areas of ischemic bowel during surgical resection. Left panel is the color image that corresponds to the tissue hemoglobin oxygenation image on the right. Blue areas in the oxygenation image show areas of the bowl that are poorly oxygenated while red areas are indicative of high tissue hemoglobin oxygenation.

\subsection{Example from peripheral vascular assessment}

Poor peripheral circulation to the lower legs, feet and toes is common in the diabetic population and the elderly. This can result in chronic wounds with limited healing potential and ultimately amputation. Non-invasive red - near infrared spectral imaging is well suited for surveying lower limb circulation to locate areas of critically low oxygenation. The technology has value in monitoring poorly healing wounds and providing an early indicator of their response to treatment. The left side of Figure 4 shows the RGB images of the left and right foot of a patient while the right panel shows the corresponding Snapshot $\mathrm{S}_{\mathrm{t}} \mathrm{O}_{2}$ image. The right foot has poor tissue hemoglobin oxygenation compared to the left and the index toe of the right foot is particularly poorly oxygenated. 

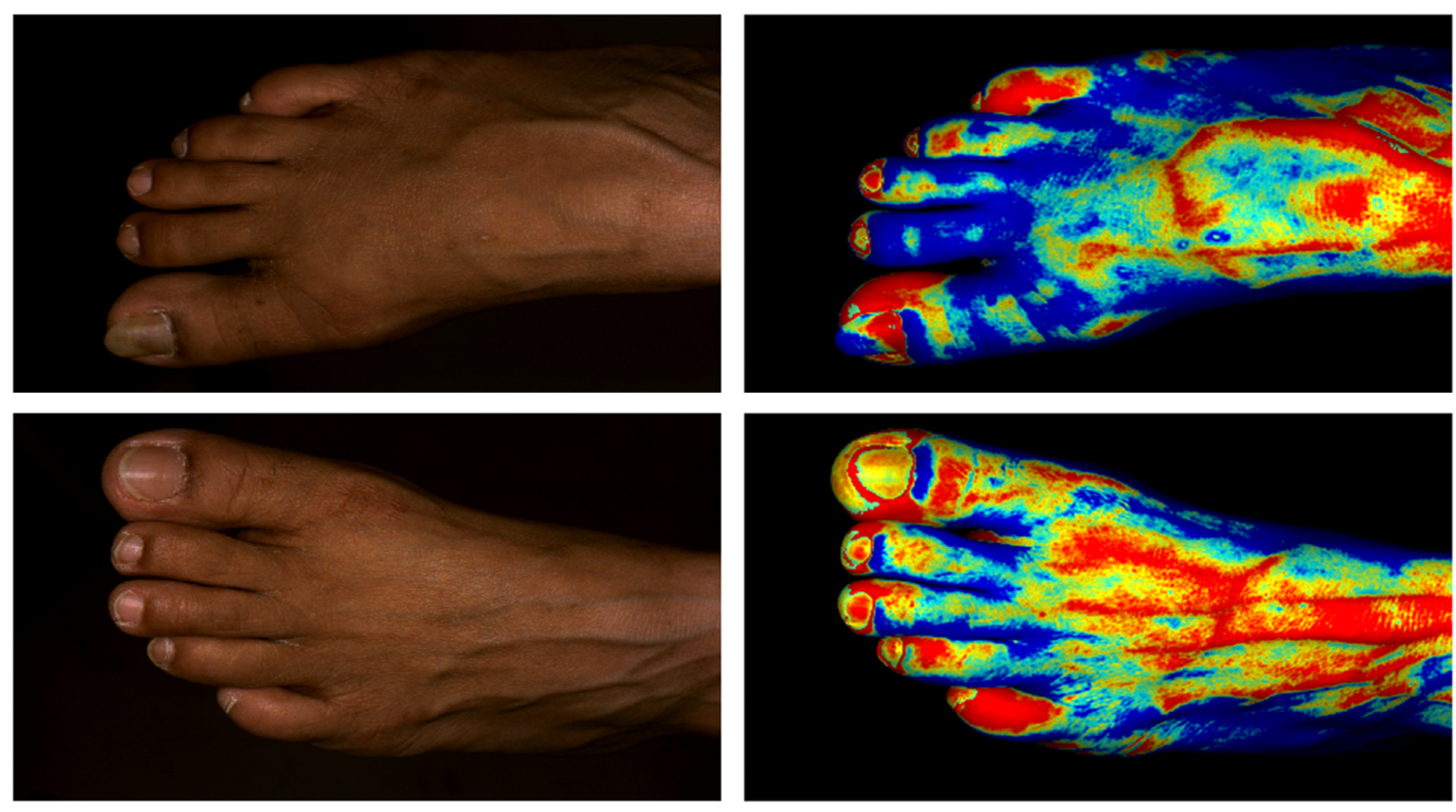

Figure 4. SnapshotNIR images of the left and right foot of a patient with suspected circulatory compromise to the toes. Left panel of images show the color digital pictures of the left and right foot that correspond to the right panel of tissue hemoglobin oxygenation images. The hemoglobin oxygenation images suggest that the index toe of the right foot of the patient is compromised.

\section{CONCLUSIONS}

The relative proportion of oxygenated to deoxygenated hemoglobin can be determined by measuring the reflected light in red and near infrared regions of the spectrum when blood perfused tissue is illuminated. Combining this method with multi spectral red - near infrared imaging enables one to capture high fidelity images of the distribution of hemoglobin oxygenation in the vascular beds that run near the surface of the tissue being imaged. Such tissue hemoglobin oxygenation images can be acquired in real time with highly portable, handheld, battery powered devices. These devices can be configured to be as convenient to use as a digital camera. These convenience factors combined with the clinically valuable information they can provide on local tissue hemoglobin oxygenation make them suitable for use in a wide range of clinical settings. We have provided specific examples in the area of general surgery and peripheral vascular assessment / chronic wound care where this type of technology could play a meaningful role.

\section{REFERENCES}

[1] Sowa M.G., Friesen J.R., "Near-infrared Spectroscopy, In Vivo Tissue Analysis by". Encyclopedia of Analytical Chemistry, 10/2013; DOI:10.1002/9780470027318.a0113.pub2

[2] Sowa M.G., Kuo W-C, Ko A. C-T, Armstrong D.G., "Review of near-infrared methods for wound assessment" JBO 01/2016; 21(9):091304.

[3] Sowa M., Cervi M, "Hybrid visible and near infrared imaging with an rgb color filter array sensor", Ref. No. W02019051591A1, Application WIPO (PCT) Year: 09/2017

[4] Jones G.E., Yoo A., King V.A., Sowa M., Pinson D.M., "Snapshot multispectral imaging is not inferior to SPY laser fluorescence imaging when predicting murine flap necrosis”, Plastic and Reconstructive Surgery (inpress) 2019. 